\title{
A new species of Panax L. (Araliaceae) from Arunachal Pradesh, India
}

\author{
Momang Taram ${ }^{1}$, A.P. Das and Hui Tag \\ Plant Systematic and Ethnobotanical Research Laboratory, Department of Botany, Rajiv Gandhi University, \\ Rono Hills, Doimukh - 791112, Arunachal Pradesh, India \\ ${ }^{1}$ Corresponding author \\ E-mail IDs: momangtaram9@gmail.com; apdas.nbu@gmail.com; huitag2008rgu@gmail.com
}

[Received 01.11.2018; Revised 09.12.2018; Accepted 14.12.2018; Published 31.12.2018]

\begin{abstract}
Panax arunachalensis, a new species in Araliaceae from Ziro valley in Lower Subansiri District of Arunachal Pradesh in Northeastern India is recognized and named. Its nomenclature along with detailed description, distribution, suitable photographs and illustration are provided. A comparison of the species with known species of Panax L. from the Indian Himalayan region is also provided.
\end{abstract}

Key words: Panax anunachalensis, New species, Araliaceae, Ziro Valley, Arunachal Pradesh, India

\section{INTRODUCTION}

Araliaceae (the ginseng family) includes about 50 genera and approximately 1500 species. The family is distributed mostly in tropics and subtropics with some genera occurring in temperate regions (e.g. Aralia, Hedera, Oplopanax and Panax) (Pandey et al. 2007). The Himalayas and central and western China are the current centre of diversity of the genus (Wen \& Zimmer 1996). The plants of the family include perennial herbs, trees, vines, epiphytes and succulents.

The systematics of Araliaceae is currently under study and taxonomic changes and novelties are to be expected (https://en.wikipedia.org/wiki/Araliaceae). Species of Araliaceae are of crucial significance in traditional Chinese medicine in which Panax L. is medicinally most important genus (Jiang et al. 2018).

The genus Panax was established by Linnaeus (1753) and was described under Pentandria digyna (Pandey et al. 2007) and is consist of 19 species world wide of which two species grow in eastern North America and other species in Eastern-Central Asia (Venugopal $\&$ Ahuja 2014). Seven species of Panax are described in China (e-flora of china) and 11 of its accepted species are listed in the plant list (www.theplantlist.org). But, Kew Science has listed 13 accepted species for the genus, which includes Panax assamicus R.N Banerjee and Panax sokpayensis Shiva K. Sharma \& Pandit (www.plantsoftheworldonline.org). All the Panax species are commonly known as ginseng and commercially its name go with their country of origin (Gurung et al. 2016). Maximum species diversity in Panax is reported from eastern, southwestern and central provinces of China, Central to Eastern Himalaya (Nepal, Bhutan and India) and some parts of South-East Asia. The lack of congruence in morphological characters among Panax species has made the taxonomic delimitation in the genus rather controversial (Sharma \& Pandit 2009). In India, Panax occurs mostly in the north and northeastern regions 

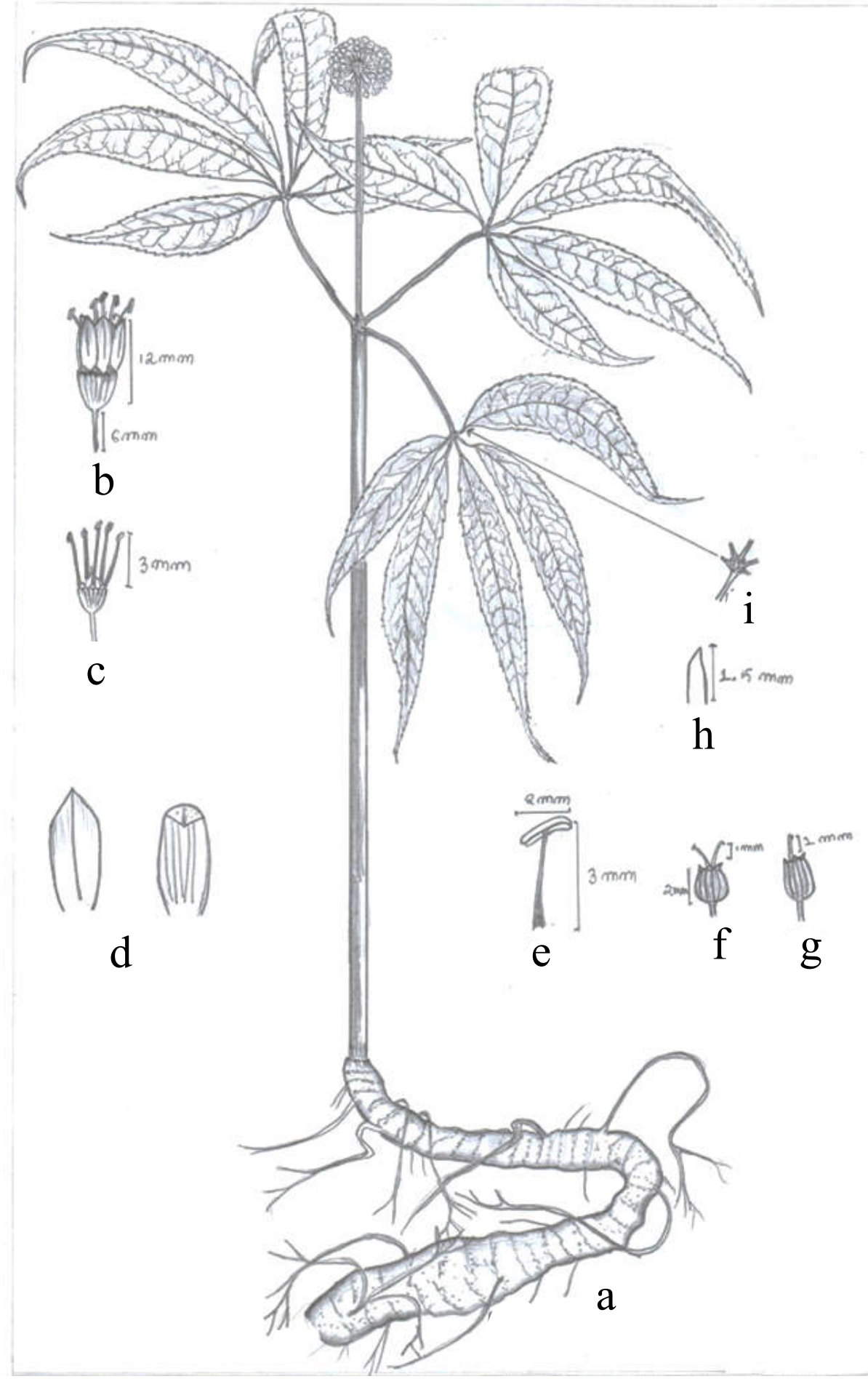

Figure 1. Illustration of Panax arunachalensis M.Taram, A.P. Das \& H.Tag: a. Plant habit; b. Single flower; c. Flower parts corolla removed; d. Dorsal and ventral surface of corolla; e. Stamen; f. Gynoecium with 2 styles; g. Gynoecium with 1 style; h. Bract; i. Stipels at the base of leaflets 
and is widely used in folk medicines (Pandey et al. 2007). Clarke (1879) reported two species, viz. Aralia pseudoginseng (Wall.) Benth. ex C.B. Clarke (syn. Panax pseudoginseng Wall.) and A. bipinnatifida C.B. Clarke (syn. P. bipinnatifidus Seem.) from Sikkim Himalaya and Banerjee (1968) described two new species, viz. P. sikkimensis R.N. Banerjee and P. assamicus R.N. Banerjee from Sikkim in addition to P. pseudoginseng and P. bipinnatifidus and, later on, Sharma and Pandit (2009) described Panax sokpayensis Sharma \& Pandit from the Sikkim Himalaya.

Taxonomic position of panax bipinnatifidus was controversial and problematic. Sharma and Pandit (2011) worked on the morphometric analysis and taxonomic study of Panax species from the Sikkim Himalaya and concluded that P. sikkimensis R.N. Banerjee is the synonym of $P$. bipinnatiûdus Seem. in Indian Himalaya.

While working on the Panax species of Lower Subansiri District, Arunachal Pradesh under a project of Board of Research in Nuclear Science on The quality enhancement of traditional anti- oxidant plants (Panax species) from Arunachal Himalayan Region, authors have collected one Panax species having solitary umbel inflorescence from the Medicinal Plant Conservation Area at Hake Tari, Ziro. After critical study and perusal of various literatures and websites, e.g. Sharma \& Pandit (2009, 2011), Pandey et al. (2007), Flora of China@, efloras.org, envis.frlht.org $>$ plantdetails, https://plants.jstor.org, apps.kew.org $>$ herbcat, and m.flowersofindia.net, it revealed that it is hitherto one undescribed species having close affinity with Panax sokpayensis Shiva K. Sharma \& Pandit and Panax assamicus R.N. Banerjee. Hence this species is described herein as a new species with illustrations and photographs.

\section{TAXONOMIC TREATMENT}

Panax arunachalensis M.Taram, A.P Das \& H.Tag, sp. nov.

Panax arunachalensis differs from Panax sokpayensis Shiva K. Sharma \& Pandit in having trichomes on both surfaces of leaf, lanceolate leaflets, Inflorescence with single terminal umbel, triangular calyx-lobes and much smaller bracts. The new species differs from Panax assamicus R.N. Banerjee in having horizontal rhizome, stem color, lanceolate leaflets with oblique base, triangular calyx, lanceolate petals, fruit having lower portion red and apical part black and flattish seed. It also differs from Panax bipinnatiûdus Seem. in having horizontal rhizome, pinnatified lobed leaflets, trichomes on both surfaces of leaf, linear bracts and flattish seeds; and differs from Panax pseudoginseng Wall. in having horizontal rhizome, stem color, lanceolate leaflets with attenuate or oblique base, presence of trichomes on both surfaces of leaf, single umbel inflorescence, linear bract, lanceolate corolla and fruits with lower portion red and only apical part black.

TYPE: INDIA, Arunachal Pradesh: Lower Subansiri District. Ziro Valley, $2300 \mathrm{~m}, 27.63^{\circ}$ N latitude $93.83^{\circ}$ E longitude, dated23.12.2017, Momang Taram, A.P Das \& Hui Tag HAU/MT-790/23.12.2017/Hake Tari (Holotype: ASSAM; Paratype: HAU, ARUN)

Perennial herbs, $40-110 \mathrm{~cm}$ tall; rhizomes $25-30 \mathrm{~cm}$, horizontal, variable in shape with prominent stem scars and rings at node; internodes short and thick, ca. $0.4-0.6 \mathrm{~cm}$. Stem stout, brownish-green, terete, erect, $24-95 \times 0.3-0.7 \mathrm{~cm}$ with scales at base. Leaves $2-$ 7 , verticillate at stem-tip, $18-30 \mathrm{~cm}$ long, exstipulate, palmately compound; petioles glabrous, stout, $8-20 \mathrm{~cm}$ long; stipels $0.013 \mathrm{~cm}-0.014 \mathrm{~cm}$, linear to lanceolate; petiolules $1-4 \mathrm{~cm}$ with different sizes on a leaf; leaflets $5-7$, lateral leaflets shorter, lanceolate, $10-25.5 \times$ $2.5-4.5 \mathrm{~cm}$, lateral one unequal-sided, serrulate, narrowly caudate-acuminate, cauda up to $4 \mathrm{~cm}$ long, base attenuate, oblique in lateral ones, both surfaces setose, trichomes $0.08 \mathrm{~cm}-$ 

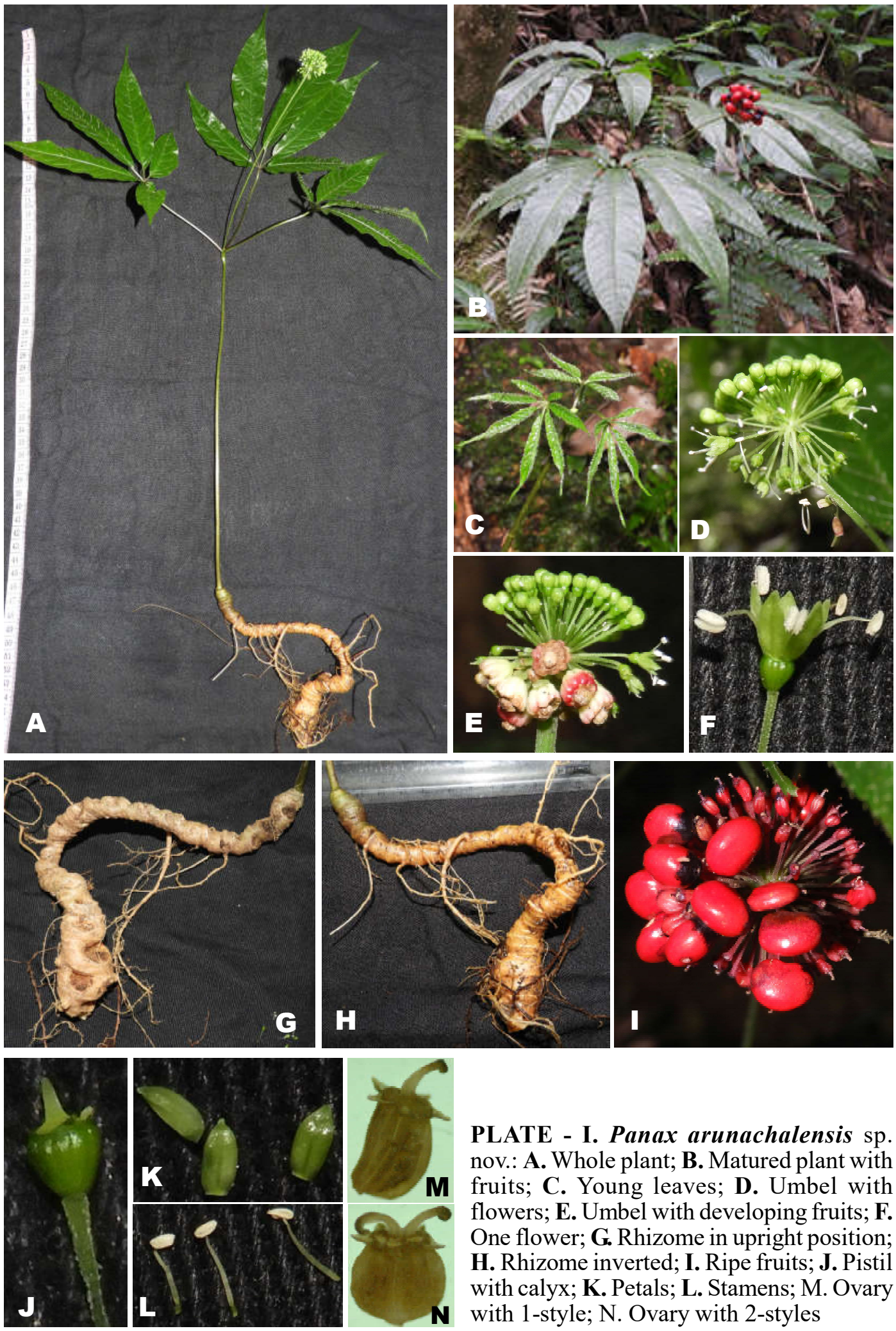

PLATE - I. Panax arunachalensis sp. nov.: A. Whole plant; B. Matured plant with

M fruits; C. Young leaves; D. Umbel with flowers; E. Umbel with developing fruits; $\mathbf{F}$. One flower; G. Rhizome in upright position; H. Rhizome inverted; I. Ripe fruits; J. Pistil with calyx; K. Petals; L. Stamens; M. Ovary with 1-style; N. Ovary with 2-styles 
$0.09 \mathrm{~cm}$ long, cylindrical on veins and veinlets; venation reticulate. Umbel solitary, simple, terminal; peduncle stout, $13-20 \mathrm{~cm}$ long; bracts linear $0.1-0.3 \times 0.1 \mathrm{~cm}$, green; pedicels $0.5-1 \mathrm{~cm}$ long, glaucous. Flowers $80-90$ per umbel, actinomorphic, bisexual, epigynous; calyx persistent, sepals 5, deltoid, glabrous, alternate to petals, green; petals 5, free, lanceolate, $0.2 \times 0.1 \mathrm{~cm}$, glabrous, yellowish-green; stamens 5 , free, $0.3-0.4 \mathrm{~cm}$, filaments free $0.3 \mathrm{~cm}$, anthers oblong, bilobed, white, ca. $0.2 \times 0.1 \mathrm{~cm}$, dorsifixed; carpels $2-3$, syncarpous, ovary inferior, glabrous, green; style 1 or 2, stigma continuous, green; placentation apical. Berry more or less reniform, lower portion red and only apical part black, ca. $0.9 \mathrm{~cm}$ broad and 1.2 cm long; seed large and flattish ca. $0.6-0.9 \mathrm{~cm}$ long.

Flowering: June - August; Fruiting: September - December

Distribution and Ecology: Geographical location of the type locality is $27.63^{\circ} \mathrm{N}$ Latitude and $93.83^{\circ}$ E Longitude. So far, Panax arunachalensis is known only from the type locality and is growing in temperate environment of $2200 \mathrm{~m}$ amsl at Ziro valley in Lower Subansiri district of Arunachal Pradesh, India. The species is shade loving, grows on moist humus-rich soil of forest floor along with Strobilanthes sp., Mycetia sp., Anoectochilus sp., etc. According to IUCN Red List categories and criteria (IUCN 2014), this species should be assessed as Critically Endangered (CR) based on the present situation, i.e. restricted geographic range of less than $2 \mathrm{~km}^{2}$ with only single location (CR: B2a) and restricted population (CR: D).

Conservation status: The known population of this new species is narrowly distributed in the Medicinal Plant Conservation Area Hake Tari, Ziro Lower Subansiri District in Arunachal Pradesh and due to its high medicinal values and demand in the market, there is continuous uncontrolled harvest of its rhizome that will surely cause decline its population structure.

Etymology: The new species epithet 'arunachalensis' is based after the name of Arunachal Pradesh, the North-East Indian and extreme east Eastern Himalayan Indian state where from the species was collected and growing with its entire known population.

Table1. Comparative morphology of Panax arunachalensis sp.nov. with the other known species of Panax L. in Eastern Himalaya

\begin{tabular}{|c|c|c|c|c|c|}
\hline Character & P. arunachalensis & P. sokpayensis & P. assamicus & P. bipinnatifidus & P. pseudoginseng \\
\hline $\begin{array}{l}\text { Plant } \\
\text { height }\end{array}$ & $40-100 \mathrm{~cm}$ & $80-130 \mathrm{~cm}$ & $70-150 \mathrm{~cm}$ & $50-100 \mathrm{~cm}$ & $30-60 \mathrm{~cm}$ \\
\hline Rhizome & $\begin{array}{l}\text { Horizontal, short } \\
\text { and thick } \\
\text { internodes }\end{array}$ & $\begin{array}{l}\text { Horizontal, } \\
\text { short and thick } \\
\text { internodes }\end{array}$ & $\begin{array}{l}\text { Small zinger like, } \\
\text { horizontal, short } \\
\text { and thick } \\
\text { internodes }\end{array}$ & $\begin{array}{l}\text { Creeping, } \\
\text { elongated, intern- } \\
\text { odes slender; } \\
\text { nodes sub-globose }\end{array}$ & $\begin{array}{l}\text { Short, vertical; } \\
\text { carrot like or } \\
\text { fascicled }\end{array}$ \\
\hline $\begin{array}{l}\text { Stem } \\
\text { color }\end{array}$ & Greenish brown & Dark brown & Typically straw & Dark brown & Light straw \\
\hline Leaf & $3-7$ foliate & $4-5$ foliate & 5 - 7 foliate & $3-6$ foliate & $3-5$ foliate \\
\hline Leaflets & $\begin{array}{l}\text { Lanceolate, long } \\
\text { caudate }\end{array}$ & $\begin{array}{l}\text { Oblanceolate to } \\
\text { narrowly elliptic }\end{array}$ & Broadly linear & $\begin{array}{l}\text { Oblong-lanceolate } \\
\text { to lanceolate or } \\
\text { ovate }\end{array}$ & $\begin{array}{l}\text { Ovobate to obovate- } \\
\text { oblong }\end{array}$ \\
\hline Base & $\begin{array}{l}\text { Attenuate or } \\
\text { oblique }\end{array}$ & $\begin{array}{l}\text { Round, } \\
\text { attenuate or } \\
\text { oblique }\end{array}$ & $\begin{array}{l}\text { Rounded, rarely } \\
\text { attenuate }\end{array}$ & $\begin{array}{l}\text { Rounded or } \\
\text { attenuate, oblique }\end{array}$ & $\begin{array}{l}\text { base angustate and } \\
\text { obtuse }\end{array}$ \\
\hline Margin & Serrulate & $\begin{array}{l}\text { Entire, serrate, } \\
\text { some biserrate }\end{array}$ & $\begin{array}{l}\text { Entire, minutely } \\
\text { uniform serrate }\end{array}$ & $\begin{array}{l}\text { Pinnatifid, lobes } \\
\text { single or doubly } \\
\text { serrate }\end{array}$ & $\begin{array}{l}\text { Entire, doubly incise } \\
\text {-serrate }\end{array}$ \\
\hline Trichome & $\begin{array}{l}\text { Setose both } \\
\text { surfaces }\end{array}$ & $\begin{array}{l}\text { Setose on } \\
\text { adaxial surface }\end{array}$ & $\begin{array}{l}\text { Setose on both } \\
\text { surfaces }\end{array}$ & $\begin{array}{l}\text { Setose on adaxial } \\
\text { surface }\end{array}$ & $\begin{array}{l}\text { Setose on adaxial } \\
\text { surface }\end{array}$ \\
\hline
\end{tabular}


New species Panax arunachalensis from Arunachal Pradesh

\begin{tabular}{|c|c|c|c|c|c|}
\hline Character & P. arunachalensis & P. sokpayensis & P. assamicus & P. bipinnatifidus & P.pseudoginseng \\
\hline $\begin{array}{l}\text { Peduncle } \\
\text { length }\end{array}$ & $\begin{array}{l}\text { Shorter or equal } \\
\text { to leaf length }\end{array}$ & $\begin{array}{l}\text { Shorter than leaf } \\
\text { length }\end{array}$ & $\begin{array}{l}\text { Equal to leaf } \\
\text { length }\end{array}$ & $\begin{array}{l}\text { Longer than } \\
\text { leaves }\end{array}$ & Shorther than leaves \\
\hline $\begin{array}{l}\text { Infloresc- } \\
\text { ence }\end{array}$ & $\begin{array}{l}\text { Umbel terminal, } \\
\text { solitary }\end{array}$ & $\begin{array}{l}\text { Umbels } 6 \text {, on } \\
\text { branches of } \\
\text { peduncle }\end{array}$ & $\begin{array}{l}\text { Umbel terminal, } \\
\text { solitary }\end{array}$ & $\begin{array}{l}\text { Umbel single or } 2 \\
-5 \text { in one cluster }\end{array}$ & $\begin{array}{l}\text { Umbels } 1 \text { - } 3 \text { on } \\
\text { branches of } \\
\text { peduncle }\end{array}$ \\
\hline Bracts & $\begin{array}{l}\text { Linear, } 0.1-0.3 \\
\mathrm{~cm}\end{array}$ & $\begin{array}{l}\text { Linear, } 0.5-2.0 \\
\mathrm{~cm}\end{array}$ & $\begin{array}{l}\text { Linear, } 0.3-1.2 \\
\mathrm{~cm}\end{array}$ & Inconspicuous & Inconspicuous \\
\hline Calyx & Triangular & Lanceolate & Lanceolate & Triangular & Sub-triangular \\
\hline Corolla & Lanceolate & Lanceolate & Oblong & $\begin{array}{l}\text { Lanceolate or } \\
\text { triangular }\end{array}$ & Triangular \\
\hline Fruits & $\begin{array}{l}\text { Berry, red with } \\
\text { only apical part } \\
\text { black }\end{array}$ & $\begin{array}{l}\text { Berry, lower } \\
\text { half red, upper } \\
\text { half black }\end{array}$ & Globose, red & $\begin{array}{l}\text { Berry, lower half } \\
\text { red, upper half } \\
\text { black }\end{array}$ & Compressed, red \\
\hline Seeds & $\begin{array}{l}\text { Flattish, } 6-9 \\
\text { mm long }\end{array}$ & $\begin{array}{l}\text { Ovoid , } 4-6 \\
\text { mm long }\end{array}$ & $\begin{array}{l}\text { Ovoid,5 - } 8 \mathrm{~mm} \\
\text { long }\end{array}$ & $\begin{array}{l}\text { Ovoid, } 5-7 \mathrm{~mm} \\
\text { long }\end{array}$ & $\begin{array}{l}\text { Flattish, } 5-8 \mathrm{~mm} \\
\text { long }\end{array}$ \\
\hline
\end{tabular}

\section{Acknowledgements}

The authors are thankful to Board of Research in Nuclear Sciences (BRNS), Department of Atomic Energy, Government of India for Funding Support through a research project. Authors are deeply thankful to Prof. A.K Pandey, Vice-Chancellor, Mansarovar Global University, Bhopal for his valuable comments. The first author is thankful to Ojar Taku for his kind help during the field work.

\section{LITERATURE CITED}

Banerjee, R.N. 1968. A Taxonomic Revision of Indian Panax L. (Araliaceae). Nelumbo 10(1). DOI: $10.20324 /$ nelumbo/v10/1968/76105

Clarke, C.B. 1879. Araliaceae. In: Hooker, J. D.(ed.), The Flora British India, vol 2, L. Reeve \& Co Ltd, Henrieta street, Covent Garden, London. Pp. 720 - 740.

Flora of China@efloras.org

Gurung, B.; Bhardwaj, K.P. \& Talukdar, N.C. 2016. Substractive transcriptome analysis of leaf and rhizome reveals differentially expressed transcripts in Panax sokpayensis. Functional and Integrative Genomics 16(6): 619 - 639.

https://cmsdata.iucn.org [IUCN Red List Guidelines, February 2014]

https://en.wikipedia.org/wiki/Araliaceae

http://envis.frlht.org>plantdetails

https://plants.jstor.org

Jiang, P.; Shi Feng-Xue; Li Ming-Rui; Liu Bao; Wen, J.; Xiao Hong-Xing and Lin Feng Li. 2018. Positive selection driving cytoplasmic genome evolution of the medicinally important ginseng plant genus Panax. Frontier in plant Science vol. 9: article 359

Linnaeus, C. 1753. Species Plantarum, ed. 1: 692. Laurentius Salvius. Sweden.

Pandey, A.K.; Ali, M.A. \& Mao, A.A. 2007. Genus Panax L. (Araliaceae) in India, Pleione 1(2): $46-54$.

Sharma, S.K. \& Pandit, M.K. 2009. A New Species of Panax L. (Araliaceae) from Sikkim Himalaya, India. Syst. Bot. 34(2): $434-438$. 
Sharma, S.K. \& Pandit, M.K. 2011. A morphometric analysis and taxonomic study of panax bipinnatifidus Seem. (Araliaceae) species complex from Sikkim Himalaya, India. $P l$. Syst. Evol. DOI 10.1007/s00606-011-0501-8.

Venugopal, N. \& Ahuja, P. 2014. Reproductive biology of Panax wangianus (Araliaceae): a critically endangered medicinal plant. Intn. J. Pl. Reprod. Biol. 6(2): $122-128$.

Wen, J. \& Zimmer, E.A. 1996. Phylogeny and Biogeography of Panax L.(The Ginseng Genus, Araliaceae):Inferences from ITS Sequences of Nuclear Ribosomal DNA. Molec. Phylogen. Evol. 6(2): 167 - 177.

www.apps.kew.org $>$ herbcat

www.m.flowersofindia.net

www.the plant list.org 